\title{
Feasibility and response of helical tomotherapy in patients with metastatic colorectal cancer
}

\author{
Sun Hyun Bae, MD, PhD¹, Seong Kwon Moon, MD, PhD1', Yong Ho Kim, MD, PhD², \\ Kwang Hwan Cho, PhD', Eung Jin Shin, MD, PhD³, Moon Sung Lee, MD, PhD', \\ Chang Beom Ryu, MD, PhD', Bong Min Ko, MD, PhD , Jina Yun, MD, $\mathrm{PhD}^{5}$ \\ 'Department of Radiation Oncology, Soonchunhyang University College of Medicine, Bucheon; \\ ${ }^{2}$ Department of Radiation Oncology, Catholic Kwandong University International St. Mary's Hospital, Incheon; \\ ${ }^{3}$ Department of General Surgery, Soonchunhyang University College of Medicine, Bucheon; \\ Divisions of ${ }^{4}$ Gastroenterology and ${ }^{5} \mathrm{Hematology-Oncology}$, Department of Internal Medicine, \\ Soonchunhyang University College of Medicine, Bucheon, Korea
}

Purpose: To investigate the treatment outcome and the toxicity of helical tomotherapy (HT) in patients with metastatic colorectal cancer (mCRC).

Materials and Methods: We retrospectively reviewed 18 patients with 31 lesions from mCRC treated with HT between 2009 and 2013. The liver (9 lesions) and lymph nodes (9 lesions) were the most frequent sites. The planning target volume (PTV) ranged from 12 to $1,110 \mathrm{~mL}$ (median, $114 \mathrm{~mL}$ ). The total doses ranged from 30 to $70 \mathrm{~Gy}$ in 10-30 fractions. When the $\alpha / \beta$ value for the tumor was assumed to be $10 \mathrm{~Gy}$ for the biologically equivalent dose (BED), the total doses ranged from 39 to $119 \mathrm{~Gy}_{10}$ (median, 55 $\mathrm{Gy}_{10}$ ). Nineteen lesions were treated with concurrent chemotherapy (CCRT).

Results: With a median follow-up time of 16 months, the median overall survival for 18 patients was 33 months. Eight lesions (26\%) achieved complete response. The 1- and 3-year local progression free survival (LPFS) rates for 31 lesions were 45\% and 34\%, respectively. On univariate analysis, significant parameters influencing LPFS rates were chemotherapy response before $H T$, aim of HT, CCRT, PTV, BED, and adjuvant chemotherapy. On multivariate analysis, PTV $\leq 113 \mathrm{~mL}$ and BED $>48 \mathrm{~Gy}_{10}$ were associated with a statistically significant improvement in LFPS. During HT, four patients experienced grade 3 hematologic toxicities, each of whom had also received CCRT.

Conclusion: The current study demonstrates the efficacy and tolerability of HT for mCRC. To define optimal RT dose according to tumor size of $m C R C$, further study should be needed.

Keywords: Colorectal cancer, Helical tomotherapy, Metastases

\section{Introduction}

Generally, for patients with metastases, the treatment trend has been toward using short courses of radiotherapy (RT) with palliative intent $[1,2]$. Advances in cancer care, including therapeutic advanced in surgery, RT techniques, and systemic therapy, however, have changed the general rules for treating metastatic cancer. Systemic therapies have extended

Received 2 November 2015, Revised 26 November 2015, Accepted 10 December 2015.

Correspondence: Seong Kwon Moon, MD, PhD, Department of Radiation Oncology, Soonchunhyang University College of Medicine, 170 Jomaru-ro, Wonmi-gu, Bucheon 14584, Korea. Tel: +82-32-621-5887, Fax: +82-32-621-5018, E-mail: drmoonrt@schmc.ac.kr

(c) This is an Open Access article distributed under the terms of the Creative Commons Attribution Non-Commercial License (http://creativecommons.org/ licenses/by-nc/4.0/) which permits unrestricted non-commercial use, distribution, and reproduction in any medium, provided the original work is properly cited.

www.e-roj.org 
overall survival (OS) times in selected patients and now an intermediate state of metastases termed "oligometastases" offers the opportunity for cure $[3,4]$. These clinical settings emphasize local control of metastatic lesions with palliativeintent RT, or sometimes curative-intent RT, and lead the application of advanced technologies such as intensitymodulated radiotherapy (IMRT) or stereotactic radiotherapy (SRT): high doses of irradiation may be delivered to tumor with more limited doses to surrounding normal tissues, allowing local control and symptomatic relief with minimal toxicity [5].

Colorectal cancer (CRC) is one of the most common malignancies. Approximately 25\% of patients present with metastases at initial diagnosis and almost $50 \%$ of patients will develop metastases [6]. Current standard treatment guidelines for metastatic CRC ( $m C R C)$ involve systemic therapy. Since the 1990s, median OS in mCRC was improved to 12 months with fluorouracil-based chemotherapy, to 18 months with the addition of oxaliplatin and irinotecan, and to approximately 30 months with the addition of molecular target agents such as bevacizumab, cetuximab, or panitumumab [7-9]. The disease can also present with solitary metastases or oligometastases. The increase of OS and a distinctive feature of oligometastases support the use of advanced RT techniques to maximize local control and minimize toxicity in patients with $\mathrm{mCRC}$. However, optimal RT technique and treatment result about $\mathrm{MCRC}$ has not been fully evaluated.

Therefore, we investigated the treatment outcome and the toxicity of helical tomotherapy $(\mathrm{HT})$, which is unique technique delivering IMRT through a continuous $360^{\circ}$ gantry rotation, in patients with $m C R C$.

\section{Materials and Methods}

\section{Patient selection}

From December 2009 to December 2013, 25 patients with $\mathrm{mCRC}$ received HT at Soonchunhyang University College of Medicine, Bucheon. We retrospectively reviewed patients' hospital charts and patients who met the following reasons were excluded from this study: 1) foreigner who returned to their own country after the end of HT (four patients); 2) incomplete $\mathrm{HT}$ duo to deterioration of general condition and patient refusal (two patients); and 3) loss of follow-up (one patient). The remaining 18 patients were included in this study. Among these, eight patients simultaneously received HT for 2 or 3 metastatic lesions: two patients received additional HT for other metastatic lesions later. One patient received 3 sessions of HT for multiple liver metastases. In these patients, evaluation of radiographic response to each lesion after HT was available, and all treatment sites (31 lesions) were independently analyzed for local control by HT. This study was approved by the Institutional Review Board of Soonchunhyang University College of Medicine, Bucheon (No. 2015-08-021).

\section{HT technique}

Computed tomography (CT) simulation with contrast medium was performed for each patient with 2-5 mm slice thickness during free breathing. All patients were immobilized with a posterior vacuum-lock body fixation device. In cases of lung or liver lesions, patients were asked to take shallow breaths and were immobilized with an anterior vacuum-sealed cover sheet to reduce respiratory movement. The simulation data were entered into a Pinnacle planning system (Philips Medical Systems, Madison, WI, USA). Gross tumor volume (GTV) was identified and contoured by using axial CT images. When necessary, magnetic resonance imaging and positron emission tomography/CT were used to delineate GTVs accurately. Generally, GTV was considered to be equal to the clinical target volume (CTV); CTVs of 2 para-aortic lymph node lesions and 2 colon lesions included elective nodal area; CTVs of 1 pleural lesion and 4 bone lesions had a generous margin to cover microscopic tumor extension. Planning target volume (PTV) was defined as GTV or CTV plus a setup margin of $5 \mathrm{~mm}$. When tumors were located in a moving organ, an additional margin of 5-10 $\mathrm{mm}$ was added to the PTV.

All regions of interest were transferred to a Tomotherapy Hi-Art II Planning System (Accuray Inc., Sunnyvale, CA, USA) for inverse treatment planning. Total prescribed doses depended on the physicians' judgment of the patients' performance, the extent of tumors, the expected morbidity, the aim of HT (curative vs. palliative), and whether or not the patient was receiving concurrent chemotherapy (CCRT). PTVs were prescribed as 30-70 Gy in 10-30 fractions. The optimized prescription aimed to deliver at least $95 \%$ of the prescribed dose to 95\% of PTVs. RT was delivered using a TomoTherapy device (TomoTherapy Inc., Madison, WI, USA). HT was delivered once daily and a megavoltage CT scan was performed to correct interfractional variations before each treatment.

\section{Failure definition and toxicity assessments}

Radiographic tumor response was evaluated using the Response Evaluation Criteria for Solid Tumors (RECIST) ver. 1.1 [10]. As various PTVs (curative vs. palliative) were applied, the further progression in the RT field was classified as local progression. Any progression regardless of the RT field was 
defined as disease progression. The follow-up period was defined as the time from the start of HT until the last followup or death. Toxicity within 3 months after the end of HT was assessed according to the Common Terminology Criteria for Adverse Events (CTCAE) ver. 4.0.

\section{Statistical analysis}

The primary endpoints were local progression free survival (LPFS), disease-progression free survival (DFS), and OS. Survival probabilities were estimated by the Kaplan-Meier method. To examine the relationships between possible prognostic parameters and LPFS, univariate analysis was conducted using log-rank test. PTV was analyzed as binary variable based on median value. Biologically equivalent dose (BED) was analyzed as binary variable with cut-off point of $48 \mathrm{~Gy}_{10}$, when $\alpha / \beta$ for the tumor was assumed to be $10 \mathrm{~Gy}$ [3]. Based on the result of univariate analysis, the significant variables were selected for the initial multivariate Cox proportional hazard regression model. The final multivariate model, identifying parameters that affected LFPS, was determined by backward selection eliminating nonsignificant variables with $p \geq 0.1$. All statistical analyses were performed using the SPSS software ver. 14.0 (SPSS Inc., Chicago, IL, USA), and two-sided $p<0.05$ was considered statistically significant.

\section{Results}

\section{Patients' and treatment characteristics}

Initially, eight patients (44\%) were diagnosed with rectal cancer and 10 patients (56\%) with colon cancer. Twelve patients (61\%) underwent resection of the primary tumor: 11 patients with curative intent and one patient with palliative intent. Six patients (39\%) received palliative chemotherapy alone. Patients' age at the time of HT for mCRC ranged from 18 to 79 years (median, 55 years). There were 9 males (50\%) and 9 females (50\%). The Eastern Cooperative Oncology Group performance scores were 1 in 17 patients (94\%) and 2 in one patient (6\%). A total of 31 lesions were treated with HT: the liver (9 lesions, 29\%) and LNs (9 lesions, 29\%) were the most frequent sites. Two lesion (7\%) presented regional recurrence and 10 lesions (32\%) presented oligometastases, which was defined as limited metastases $\leq 5$ on image study. These 12 lesions were treated with curative-intent $\mathrm{HT}$, which signified the inclusion of all viable tumors within the RT field. On the other hand, 19 lesions (61\%) presented distant metastases and were treated with palliative-intent. Five lesions (16\%) received reirradiation (Re-RT): 1 pelvic LN and 1 seeding nodule were
Table 1. Treatment characteristics

\begin{tabular}{|c|c|}
\hline Parameter & No. of lesions \\
\hline \multicolumn{2}{|l|}{ Treatment site } \\
\hline Lung & 3 \\
\hline Liver & 9 \\
\hline Lymph nodes & 9 \\
\hline Bone & 5 \\
\hline Colon & 2 \\
\hline Seeding nodule & 2 \\
\hline Pleura & 1 \\
\hline \multicolumn{2}{|l|}{ Disease status } \\
\hline Regional recurrence & 2 \\
\hline Oligometastases $^{\mathrm{a})}$ & 10 \\
\hline Distant metastases & 19 \\
\hline \multicolumn{2}{|l|}{ Aim of HT } \\
\hline Curative & 12 \\
\hline Palliative & 19 \\
\hline \multicolumn{2}{|l|}{ Chemotherapy response before HT } \\
\hline Upfront radiotherapy & 10 \\
\hline PR after chemotherapy & 2 \\
\hline SD after chemotherapy & 4 \\
\hline PD after chemotherapy & 15 \\
\hline \multicolumn{2}{|l|}{ Reirradiation } \\
\hline Yes & 5 \\
\hline No & 26 \\
\hline \multicolumn{2}{|l|}{ CCRT } \\
\hline Yes & 19 \\
\hline No & 12 \\
\hline PTVs (mL), median (range) & $114(12-1,100)$ \\
\hline Total dose (Gy), median (range) & $44(30-70)$ \\
\hline $48(1.6 \mathrm{~Gy} / f x)$ & 2 \\
\hline 51 (1.7 Gy/fx) & 3 \\
\hline $40(2.0 \mathrm{~Gy} / f x)$ & 4 \\
\hline $42(2.1 \mathrm{~Gy} / \mathrm{fx})$ & 2 \\
\hline 44 (2.2 Gy/fx) & 5 \\
\hline 45 (2.25 Gy/fx) & 1 \\
\hline 69 (2.3 Gy/fx) & 1 \\
\hline $50(2.5 \mathrm{~Gy} / f x)$ & 3 \\
\hline 30 \&t 33 (3.0 Gy/fx) & 2 \\
\hline 35 (3.5 Gy/fx) & 2 \\
\hline 40 (4.0 Gy/fx) & 1 \\
\hline $50(5.0 \mathrm{~Gy} / \mathrm{fx})$ & 3 \\
\hline 70 (7.0 Gy/fx) & 2 \\
\hline BED $\left(\mathrm{Gy}_{10}\right)$, median (range) & $55(39-119)$ \\
\hline \multicolumn{2}{|l|}{ Adjuvant chemotherapy } \\
\hline Yes & 18 \\
\hline No & 13 \\
\hline
\end{tabular}

$H T$, helical tomotherapy; PR, partial response; SD, stable disease; $P D$, progressive disease; CCRT, concurrent chemoradiotherapy; PTV, planning target volume; BED, biologically equivalent dose when $\alpha / \beta$ for the tumor was assumed to be $10 \mathrm{~Gy}$.

a) Limited metastases $\leq 5$ on image study. 
overlapped with previous preoperative CCRT; 2 liver lesions were overlapped with previous HT to liver metastases; 1 pelvic LN was consulted duo to PD after previous palliative RT with 2-dimensional (2D) technique. Remaining treatment characteristics are summarized in Table 1. PTV and BED according to RT site are shown in Fig. 1.

\section{Treatment outcomes}

The median follow-up from HT start date was 16 months (range, 2 to 54 months): five-alive patients were follow-up loss within 1 year. The median survival time of 18 patients was 33 months, and the OS rate was $68 \%$ and $42 \%$ at 1 and 3 years, respectively. The DFS rate was $22 \%$ and $11 \%$ at 1 and 3

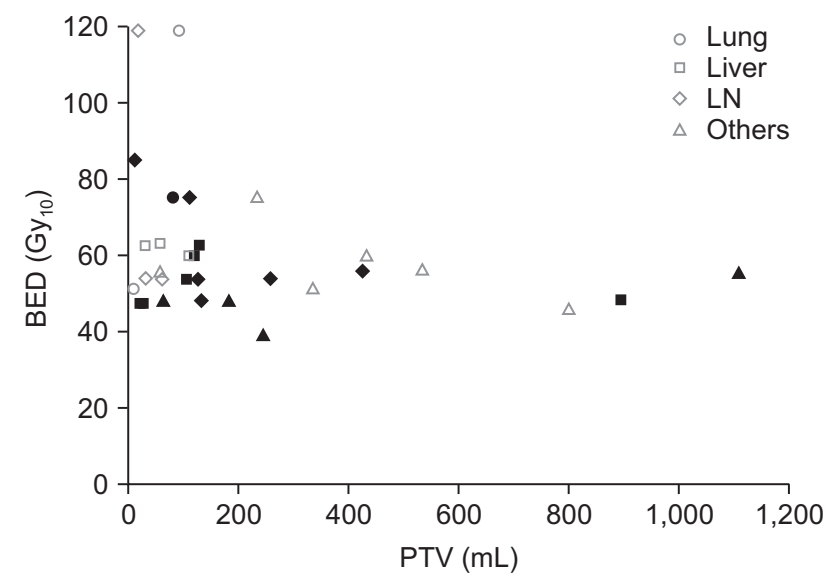

Fig. 1. Planning target volumes (PTV) and biologically equivalent dose (BED) according to treatment site. Grey color indicates lesion without local progression; black color with local progression; others include bone, colon, seeding nodule, and pleural metastases. LN, lymph node.

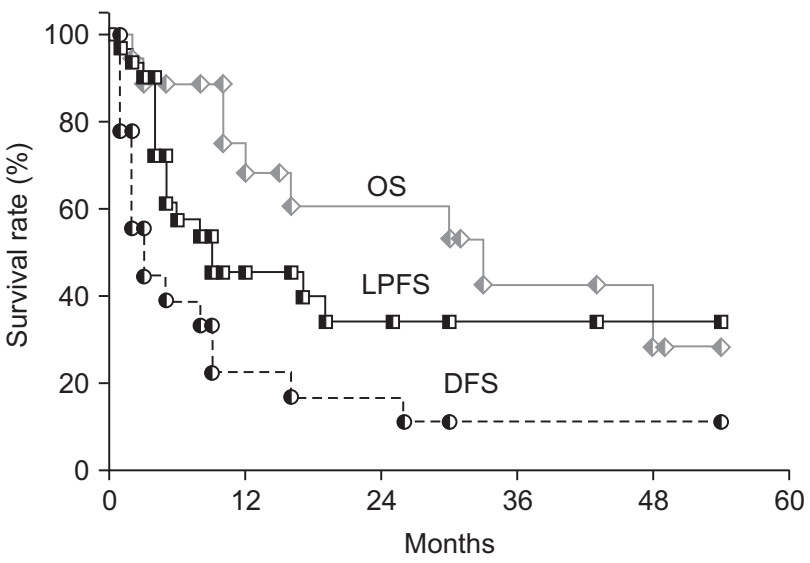

Fig. 2. Local progression-free survival (LPFS), disease-free survival (DFS), and overall survival (OS) after helical tomotherapy for metastatic colorectal cancer. years, respectively. Among 31 lesions, complete response (CR) was achieved in 8 lesions (26\%) at 2-12 months after HT: CR of 2 colon lesions was confirmed by pathologic review. The LPFS rate for 31 lesions was 45\% and 34\% at 1 and 3 years, respectively. Details regarding survival outcomes are shown in Fig. 2.

On univariate analysis, chemotherapy response before $\mathrm{HT}_{\text {, }}$ aim of HT, CCRT, PTV, BED, and adjuvant chemotherapy were significant parameters in determining LPFS for 31 lesions (Table 2). On Cox multivariate regression analysis, PTV ( $\leq 113 \mathrm{~mL}$ vs. $>113 \mathrm{~mL}$; hazard ratio [HR], 5.155; 95\% confidence interval [Cl], 1.697 to $15.661 ; p=0.004)$ and BED ( $\leq 48 \mathrm{~Gy}_{10}$ vs. $>48$ $\mathrm{Gy}_{10} ; \mathrm{HR}, 4.539 ; 95 \% \mathrm{Cl}, 1.492$ to $13.816 ; \mathrm{p}=0.008$ ) were statistically significant factors affecting LPFS. Fig. 3 shows

Table 2. Univariate analysis for parameters affecting LPFS

\begin{tabular}{|c|c|c|c|}
\hline Parameter & $\begin{array}{l}\text { No. of } \\
\text { lesions }\end{array}$ & $\begin{array}{c}3-y r \text { LPFS } \\
(\%)\end{array}$ & p-value \\
\hline Radiotherapy site & & & 0.680 \\
\hline Lung & 3 & 67 & \\
\hline Liver & 9 & $33^{\text {a) }}$ & \\
\hline Lymph nodes & 9 & 21 & \\
\hline Others ${ }^{\mathrm{b})}$ & 10 & $32^{\mathrm{a})}$ & \\
\hline Chemotherapy response before HT & & & 0.000 \\
\hline Progressive disease & 15 & 0 & \\
\hline Others ${ }^{c)}$ & 16 & 58 & \\
\hline Aim of HT & & & 0.018 \\
\hline Curative & 12 & 56 & \\
\hline Palliative & 19 & 0 & \\
\hline Reirradiation & & & 0.160 \\
\hline Yes & 5 & 20 & \\
\hline No & 26 & 36 & \\
\hline CCRT & & & 0.007 \\
\hline Yes & 19 & 53 & \\
\hline No & 12 & 0 & \\
\hline Planning target volume & & & 0.002 \\
\hline$\leq 113 \mathrm{~mL}$ & 15 & 51 & \\
\hline$>113 \mathrm{~mL}$ & 16 & $16^{a)}$ & \\
\hline BED & & & 0.005 \\
\hline$\leq 48 \mathrm{~Gy}_{10}$ & 8 & 0 & \\
\hline$>48 \mathrm{~Gy}_{10}$ & 23 & 48 & \\
\hline Adjuvant chemotherapy & & & 0.007 \\
\hline Yes & 18 & 58 & \\
\hline No & 13 & 0 & \\
\hline
\end{tabular}

LPFS, local progression free survival; HT, helical tomotherapy; CCRT, concurrent chemoradiotherapy; BED, biologically equivalent dose when $\alpha / \beta$ for the tumor was assumed to be $10 \mathrm{~Gy}$.

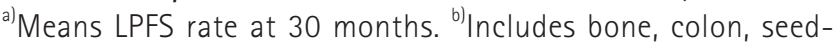
ing nodule, and pleural metastases. ${ }^{c}$ Includes partial response or stable disease after chemotherapy before HT or upfront RT when distant metastases are developed. 
LPFS rates according to PTV and BED.

\section{Toxicities}

Grade 3 hematologic toxicity was occurred in four patients during HT (Table 3). They received CCRT: two patients (patients 1 and 4 in Table 3) received further chemotherapy with a dose reduction of $80 \%$. On the other hand, there was no nonhematologic toxicity $\geq$ grade 3 during HT. After the end of $H T$, grade 3 gastrointestinal toxicity was reported in three patients. One patient (patient 1 in Table 3) experienced grade 3 colitis at 1 month after the end of HT; one patient (patient 2 in Table 3) experienced grade 3 stomatitis and esophagitis at 1 week after HT: gastrointestinal toxicities were occurred in an organ far from the RT field shortly after the end of HT. On the other hand, one patient received Re-RT to pelvic LN with 50 Gy in 10 fractions after 3 months following 2D RT with 30 Gy in 10 fraction and experienced grade 3 radiation-colitis at 2 months after HT. These patients with grade 3 toxicity required hospitalization and total parenteral nutrition. There was no treatment-related death.

A

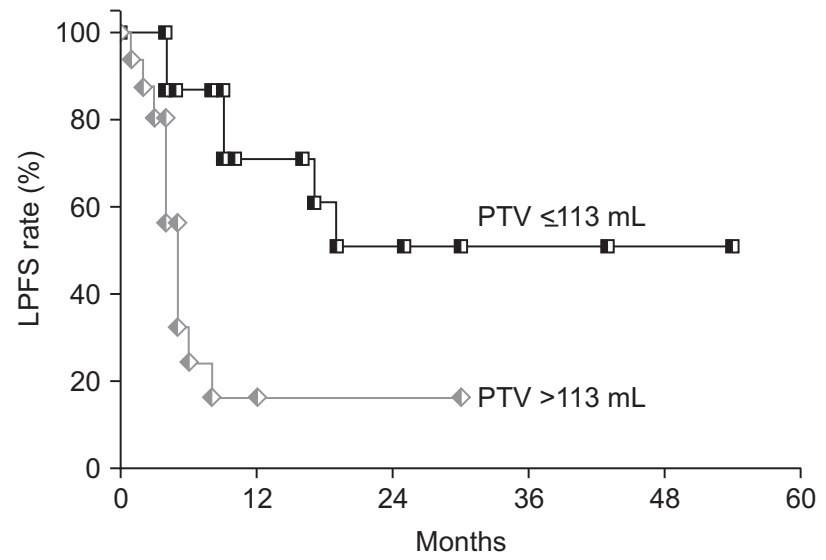

\section{Discussion and Conclusion}

The diagnosis of $m C R C$ dose not equal an acute fatal illness and might be classified more as a chronic disease with a clinical disease state between locoregionally confined, oligometastatic, and widely spread metastatic disease [11]. Several international guidelines recommend various treatment modalities according to disease status of $\mathrm{mCRC}$, although systemic therapy is mainly considered [12]. Traditionally, we apply palliative-intent RT using simple technique for mCRC. However, technical evidence, derived from IMRT's ability to deliver higher doses to the tumor, reduce toxicity, and improve quality of life $(\mathrm{QOL})$, has been used to support the adoption and implementation of IMRT for metastatic disease [13]. The Surveillance, Epidemiology and End Results-Medicare linked databases showed a significant decrease in the portion of $2 \mathrm{D}$ $R T$ and a significant increase in the use of IMRT and SRT in the last year of life among patients diagnosed with metastatic cancer [14]. HT is a unique IMRT machine, with a different dose delivery and treatment plan prescription compared

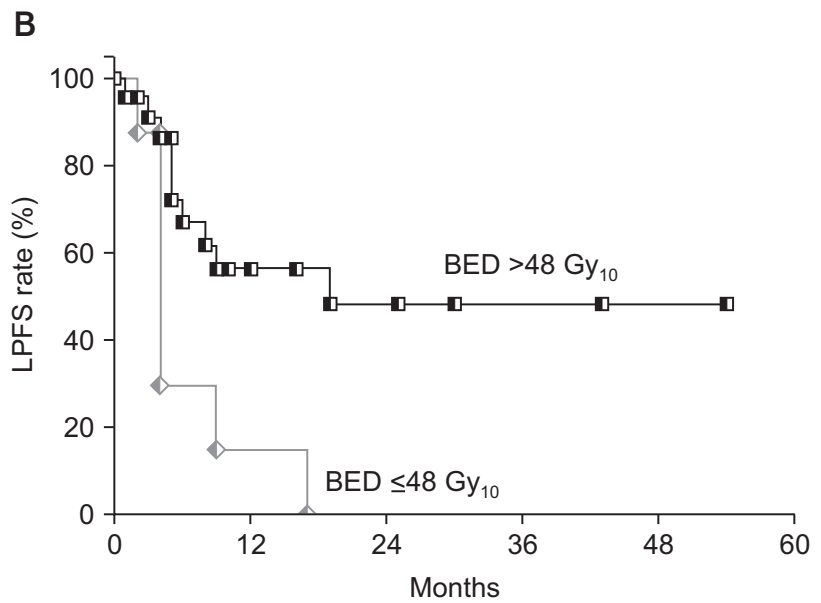

Fig. 3. (A) Local progression-free survival (LPFS) according to planning target volume (PTV) after helical tomotherapy (HT) for metastatic colorectal cancer (mCRC) on univariate analysis. (B) LPFS according to biologically equivalent dose (BED) after HT for mCRC on univariate analysis. BED was calculated when the $\alpha / \beta$ value for the tumor was assumed to be $10 \mathrm{~Gy}$.

Table 3. Hematologic toxicity during helical tomotherapy

\begin{tabular}{cclcllcl}
\hline No. & Age/sex & \multicolumn{1}{c}{ Treatment site } & PTV $(\mathrm{mL})$ & CCRT & Regimen & Grade $^{\text {a) }}$ & Adverse event \\
\hline 1 & $38 / F$ & Pleura & 1,110 & Yes & Cisplatin & 3 & Leukopenia and thrombocytopenia \\
2 & $43 / F$ & Pelvic LN & 34 & Yes & FOLFIRI & 3 & Leukopenia \\
3 & $18 / \mathrm{M}$ & Mediastinal and pelvic LN & 259 \& 63 & Yes & FP & 3 & Leukopenia \\
4 & $52 / F$ & Rib and liver & $65 \& 896$ & Yes & PLF & 3 & Thrombocytopenia \\
\hline
\end{tabular}

PTV, planning target volume; CCRT, concurrent chemoradiotherapy; LN, lymph node; FOLFIRI, irinotecan/leucovorin/fluorouracil; FP, fluorouracil/cisplatin; PLF, cisplatin/leucovorin/fluorouracil.

${ }^{a)}$ Grade was scored according to the Common Terminology Criteria for Adverse Events ver. 4.0. 
to conventional linear accelerators-based IMRT, enabling treatment implementation for a wide spectrum of clinical cases, ranging from very small tumor to vast target volumes [15]. A few studies of HT in patients with metastatic cancer have reported effectiveness with a symptom control rates of 76\%-90\% within a time frame as short as 6 months [16-18]. In terms of colorectal oligometastases, several studies have reported CR rates of 16\%-35\% and 1-year LPFS rates of 54\%$87 \%$ after HT [11,19-21]. The current study yielded comparable results, with a CR rate of 26\% and an LPFS rate of $45 \%$ at 1 year with median follow-up of 16 months.

In our study, PTV was the most significant parameter for LPFS on multivariate analysis. Although we agree with the necessity of further studies to improve local control of largesized colorectal oligometastases, it has been debatable in case of palliative-intent RT because of treatment related morbidity and poor OS $[21,22]$. A recent phase III study of non-small cell lung cancer reported that palliative CCRT with $42 \mathrm{~Gy}$ in 15 fractions significantly increased OS and OOL compared to chemotherapy alone, especially in tumor sizes $>7 \mathrm{~cm}$ [23]. They concluded that large tumor should not be considered a negative predictive factor in patients with poor prognosis, and patients with tumor $>7 \mathrm{~cm}$ apparently benefit from addition of RT to palliative chemotherapy. Therefore, efforts to improve local control of large tumor are needed. The other significant parameter in our study, BED, might provide some clues. Several studies have reported a dose-response relationship between RT dose and LPFS in mCRC but an optimal RT dose would not be determined [21,22,24-26]. We speculate several reasons for these variations in RT dose. First, there are interfractional and intrafractional variations of tumors located in sites that are highly affected by respiratory motion, such as the liver and the lung. If motion management is inaccurate, tumors are at high risk of being underdosed by displacements of the tumor relative to the dose distribution, especially at the border, which could finally result in a marginal recurrence regardless of total doses [27]. Second, there are differences in the radiation sensitivity of tumors. For example, Ahmed et al. [28] suggested that radiation sensitivity between primary and metastatic tissues of colon cancer revealed significant differences based on anatomical location of metastases. Therefore, further studies are necessary to identify optimal RT dose according to radiation sensitivity of $\mathrm{mCRC}$ under a thorough control of moving organ.

During HT, four patients experienced grade 3 hematologic toxicity. They received CCRT. These toxicities were partially caused by HT to large metastatic lesions. However, the effect of size of PTV might be less than anticipated, considering that the percentage of red marrow within the RT field was an independent risk factor associated with hematologic toxicity and RT sites in these patients captured about 10\% of active bone marrow in the adult $[17,29]$. In particular, the fact that two patients experienced grade 3 gastrointestinal toxicity in an organ far from the RT field shortly after the end of HT underscores the morbidity of combined treatment. Therefore, we consider CCRT in patients with good performance status, who tolerate combined modalities. Among four patients with 5 lesions who received Re-RT, 1 patient with pelvic LN metastases experienced grade 3 radiation-colitis. Considering Re-RT, we should be concerned with two points. First, acute responding tissue need some time to recover from radiation injury, and tolerance dose would be different according to time interval between initial RT and Re-RT. One study recommended a Re-RT dose after initial RT with median $50 \mathrm{~Gy}$ according to the interval as follows: 35 Gy for an interval of 3-12 months, 40-45 Gy for 12-24 months, 45-50 Gy for 24-36 months, and 50-55 Gy for more than 36 months [30]. Second, for patients with curative intent or for patients with palliative intent with a relatively long life expectancy, with the aim of durable local control, hyperfractionated Re-RT is recommended to minimize late complications [31]. Therefore, we should individually select the use of Re-RT, considering the time interval between RT sessions, the number and size of fractions, and expected morbidity and survival.

Our study has some limitations. First, this study was retrospective analysis. Thus, selection bias may have occurred, and the results may be affected. Treatment related toxicity may have been underestimated. Second, the small and heterogeneous patients' group was included, although this study was one of the few studies to address long-term results of HT confined to mCRC. To conclude the effectiveness and safety of HT to $\mathrm{mCRC}$, especially in cost effectiveness, largeprospective studies would be needed.

In conclusions, the current study demonstrates that HT for $m C R C$ is effective in durable local control: LPFS rates for 31 lesions were $45 \%$ and $34 \%$ at 1 and 3 years, respectively. To define optimal RT dose and improve the local control according to tumor size of $\mathrm{mCRC}$, further study should be needed. Patients were tolerable to HT, but for patients with CCRT the risk of toxicity showed a tendency to increase. Therefore, CCRT might be considered in patients with good performance status who can tolerate combined modalities. 


\section{Conflict of Interest}

No potential conflict of interest relevant to this article was reported.

\section{Acknowledgments}

This work was supported by the Soonchunhyang University Research Fund.

\section{References}

1. Fairchild A, Barnes $E$, Ghosh $S$, et al. International patterns of practice in palliative radiotherapy for painful bone metastases: evidence-based practice? Int J Radiat Oncol Biol Phys 2009;75:1501-10.

2. Chow E, Zeng L, Salvo N, Dennis K, Tsao M, Lutz S. Update on the systematic review of palliative radiotherapy trials for bone metastases. Clin Oncol (R Coll Radiol) 2012;24:112-24.

3. Lutz ST, Jones J, Chow E. Role of radiation therapy in palliative care of the patient with cancer. J Clin Oncol 2014;32:2913-9.

4. Hellman S, Weichselbaum RR. Oligometastases. J Clin Oncol 1995;13:8-10.

5. Jones JA, Simone CB 2nd. Palliative radiotherapy for advanced malignancies in a changing oncologic landscape: guiding principles and practice implementation. Ann Palliat Med 2014; 3:192-202.

6. Van Cutsem E, Nordlinger B, Cervantes A; ESMO Guidelines Working Group. Advanced colorectal cancer: ESMO Clinical Practice Guidelines for treatment. Ann Oncol 2010;21 Suppl 5:v93-7.

7. Cheng YD, Yang $H$, Chen GQ, Zhang ZC. Molecularly targeted drugs for metastatic colorectal cancer. Drug Des Devel Ther 2013;7:1315-22.

8. Grothey A, Sugrue MM, Purdie DM, et al. Bevacizumab beyond first progression is associated with prolonged overall survival in metastatic colorectal cancer: results from a large observational cohort study (BRiTE). J Clin Oncol 2008;26:5326-34.

9. Loupakis F, Cremolini C, Masi G, et al. Initial therapy with FOLFOXIRI and bevacizumab for metastatic colorectal cancer. N Engl J Med 2014;371:1609-18.

10. Eisenhauer EA, Therasse $P$, Bogaerts J, et al. New response evaluation criteria in solid tumours: revised RECIST guideline (version 1.1). Eur J Cancer 2009;45:228-47.

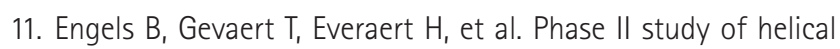
tomotherapy in the multidisciplinary treatment of oligometastatic colorectal cancer. Radiat Oncol 2012;7:34.

12. Nielsen LB, Wille-Jorgensen P. National and international guidelines for rectal cancer. Colorectal Dis 2014;16:854-65.
13. Bak K, Dobrow MJ, Hodgson D, Whitton A. Factors affecting the implementation of complex and evolving technologies: multiple case study of intensity-modulated radiation therapy (IMRT) in Ontario, Canada. BMC Health Serv Res 2011;11:178.

14. Guadagnolo BA, Huo J, Liao KP, Buchholz TA, Das P. Changing trends in radiation therapy technologies in the last year of life for patients diagnosed with metastatic cancer in the United States. Cancer 2013;119:1089-97.

15. Piotrowski T, Skorska M, Jodda A, et al. Tomotherapy: a different way of dose delivery in radiotherapy. Contemp Oncol (Pozn) 2012;16:16-25.

16. Samant $R$, Gerig $L$, Montgomery $L$, et al. The emerging role of IG-IMRT for palliative radiotherapy: a single-institution experience. Curr Oncol 2009;16:40-5.

17. Lee IJ, Seong J, Lee CG, et al. Early clinical experience and outcome of helical tomotherapy for multiple metastatic lesions. Int J Radiat Oncol Biol Phys 2009;73:1517-24.

18. Choi Y, Kim JW, Lee IJ, Han HJ, Baek J, Seong J. Helical tomotherapy for spine oligometastases from gastrointestinal malignancies. Radiat Oncol J 2011;29:219-27.

19. Kim JY, Kay CS, Kim YS, et al. Helical tomotherapy for simultaneous multitarget radiotherapy for pulmonary metastasis. Int J Radiat Oncol Biol Phys 2009;75:703-10.

20. Engels $B$, Everaert $H$, Gevaert $T$, et al. Phase II study of helical tomotherapy for oligometastatic colorectal cancer. Ann Oncol 2011;22:362-8.

21. Lee JH, Lee JH, Jang HS, et al. Hypofractionated radiotherapy with tomotherapy for patients with hepatic oligometastases: retrospective analysis of two institutions. Clin Exp Metastasis 2013;30:643-50.

22. Bae SH, Kim MS, Cho CK, et al. High dose stereotactic body radiotherapy using three fractions for colorectal oligometastases. J Surg Oncol 2012;106:138-43.

23. Strom HH, Bremnes RM, Sundstrom SH, Helbekkmo N, Aasebo U. Poor prognosis patients with inoperable locally advanced NSCLC and large tumors benefit from palliative chemoradiotherapy: a subset analysis from a randomized clinical phase III trial. J Thorac Oncol 2014;9:825-33.

24. Comito T, Cozzi L, Clerici E, et al. Stereotactic Ablative Radiotherapy (SABR) in inoperable oligometastatic disease from colorectal cancer: a safe and effective approach. BMC Cancer 2014;14:619.

25. Bae SH, Park W, Choi DH, et al. Palliative radiotherapy in patients with a symptomatic pelvic mass of metastatic colorectal cancer. Radiat Oncol 2011;6:52.

26. Cameron MG, Kersten C, Vistad I, Fossa S, Guren MG. Palliative pelvic radiotherapy of symptomatic incurable rectal cancer: a systematic review. Acta Oncol 2014;53:164-73.

27. Van den Begin $R$, Engels $B$, Gevaert T, et al. Impact of inadequate respiratory motion management in SBRT for oligometastatic 
colorectal cancer. Radiother Oncol 2014;113:235-9.

28. Ahmed KA, Fulp WJ, Berglund $A E$, et al. Differences between colon cancer primaries and metastases using a molecular assay for tumor radiation sensitivity suggest implications for potential oligometastatic SBRT patient selection. Int J Radiat Oncol Biol Phys 2015;92:837-42.

29. Ellis RE. The distribution of active bone marrow in the adult.
Phys Med Biol 1961;5:255-8.

30. Mohiuddin M, Marks G, Marks J. Long-term results of reirradiation for patients with recurrent rectal carcinoma. Cancer 2002;95:114450.

31. Guren $M G$, Undseth $C$, Rekstad $B L$, et al. Reirradiation of locally recurrent rectal cancer: a systematic review. Radiother Oncol 2014;113:151-7. 